\title{
Incorporating Event Risk into Value-at-Risk
}

\author{
Michael S. Gibson*
}

February 2001

* Trading Risk Analysis Section, Division of Research and Statistics, Federal Reserve Board. I would like to thank (without implying that they share my opinions) many Fed colleagues for stimulating discussions on the issues of specific risk and event risk and for comments on an earlier draft of this paper. I would also like to thank Adam Sanjurjo for diligent research assistance. This paper represents the views of the author and should not be interpreted as reflecting the views of the Board of Governors of the Federal Reserve System or other members of its staff. I can be reached via email at michael.s.gibson@frb.gov. Postal address: Mail Stop 91, 20th and C Streets NW, Washington, DC 20551. Phone: 1-202-452-2495. Fax: 1-202-452-3819. 


\section{Incorporating Event Risk into Value-at-Risk}

Abstract: Event risk is the risk that a portfolio's value can be affected by large jumps in market prices. Event risk is synonymous with "fat tails" or "jump risk". Event risk is one component of "specific risk," defined by bank supervisors as the component of market risk not driven by market-wide shocks. Standard Value-at-Risk (VaR) models used by banks to measure market risk do not do a good job of capturing event risk. In this paper, I discuss the issues involved in incorporating event risk into VaR.

To illustrate these issues, I develop a VaR model that incorporates event risk, which I call the Jump-VaR model. The Jump-VaR model uses any standard VaR model to handle "ordinary" price fluctuations and grafts on a simple model of price jumps. The effect is to "fatten" the tails of the distribution of portfolio returns that is used to estimate VaR, thus increasing VaR. I note that regulatory capital could rise or fall when jumps are added, since the increase in VaR would be offset by a decline in the regulatory capital multiplier on specific risk from 4 to 3.

In an empirical application, I use the Jump-VaR model to compute VaR for two equity portfolios. I note that, in practice, special attention must be paid to the issues of correlated jumps and double-counting of jumps. As expected, the estimates of VaR increase when jumps are added. In some cases, the increases are substantial. As expected, VaR increases by more for the portfolio with more specific risk.

Keywords: specific risk, market risk, jump risk, jump diffusion, default risk 


\section{Introduction}

Value-at-Risk (VaR) is a popular technique used to measure a portfolio's market risk. It identifies the loss that could occur due to fluctuations in market prices. For example, a one-day 99 percent $\mathrm{VaR}$ is the loss that would be expected to be equalled or exceeded on one out of one hundred days. VaR has been widely adopted by financial firms and bank supervisors as an important tool for risk measurement. ${ }^{1}$

"Event risk" is defined here as the risk that a market price can jump, for example due to news of a default or earnings surprise. Put simply, "event risk" and "jump risk" are the same thing. When calculating a statistical measure of market risk like VaR, there is no need to understand why a jump in the market price occurs. Only the statistical properties of the price jumps themselves are relevant. For this reason it is unnecessary to separate out subcomponents of event risk, such as the risk of an unexpected credit rating downgrade, for special treatment in a VaR model.

Few VaR models do a good job of capturing event risk. This failing is a consequence of simplifying assumptions that make it easier to compute VaR.

When bank supervisors chose to allow banks to use their internal VaR models as the basis for a capital charge for market risk, they recognized that few VaR models accurately capture event risk. They required special treatment for the component of VaR that is not driven by market-wide shocks, which they called "specific risk." "Specific risk" includes idiosyncratic risk (price fluctuations that are not correlated with market-wide shocks) and event risk. The regulatory capital charge for market risk equals

$$
3 \times \text { VaR from market-wide shocks }+4 \times \text { specific risk VaR. }
$$

The higher multiplier on specific risk VaR recognizes that VaR models typically underestimate specific risk because they do not incorporate event risk. If a bank's VaR model does capture event risk, the specific risk multiplier can be reduced to $3 \times$ at the discretion of the bank's supervisor.

In this paper, I develop a methodology to incorporate event risk into VaR. My goal is to illustrate the issues involved in adding event risk into a VaR model. Of course, the model I present is only one example of how this can be done. Still, the issues I discuss are the same ones that any model of event risk must address.

\section{VaR models}

The quality of a VaR model depends on its distributional assumption and its valuation model. By "distributional assumption," I mean what the VaR model assumes about the distribution of the underlying market risk factors upon which the portfolio's value depends. By "valuation model," I mean how the VaR model computes the portfolio's value for different shocks to market risk factors.

\footnotetext{
${ }^{1}$ Jorion (2000) provides an introduction to VaR.
} 
A good distributional assumption is one that captures the characteristics of the actual distribution of market risk factors. Table 1 lists four common distributional assumptions in VaR models. ${ }^{2}$ One important characteristic of actual data is time-varying volatility. The distributions listed in Table 1 can accommodate time-varying volatility by over-weighting data from recent history.

A second characteristic of actual market data is "fat tails." A probability distribution with "fat tails" has a greater probability mass out in the tails of the distribution, where large price moves occur, compared with the normal distribution. Some of the fat tails in actual data comes from time-varying volatility itself. However, actual market data are typically found to have fatter tails than would come from time-varying volatility alone. ${ }^{3}$ To match actual market data, an additional source of fat tails is needed, such as price jumps. In other words, price jumps, event risk and fat tails are different names for the same phenomenon. ${ }^{4}$ Note that, because large price jumps are rare events, event risk/fat tails must be estimated from a long data history.

Simultaneously capturing time-varying volatility and fat tails is a challenge. Most VaR models choose to do a good job capturing the former at the expense of the latter. Typically, VaR models over-weight recent data to capture time-varying volatility. This has the unwanted side effect of shortening the sample size, making it impossible to accurately capture the fat tails in the data. ${ }^{5}$

What is needed is a model that can combine time-varying volatility with event risk. The two components would necessarily be estimated with different data, with the time-varying volatility component using recent data and the event risk component using a long time series to accurately capture the risk of a rare event. In the next section, I present such a model, which I call the Jump-VaR model. The Jump-VaR model is probably the simplest model that fits the requirements to incorporate time-varying volatility with fat tails and event risk.

The Jump-VaR model focuses on the distribution the market risk factors are assumed to follow. I will not address the choice of "valuation model." I assume that an accurate approximation of each instrument's value as a function of market risk factor shocks is available. ${ }^{6}$

${ }^{2}$ Pritsker (1997) and Linsmeier and Pearson (2000) describe and compare various VaR models.

${ }^{3}$ See Bollerslev (1987) for some evidence.

${ }^{4}$ As a consequence, methods that explicitly account for fat tails, such as extreme value theory, are in principle capable of capturing event risk. However, it is unclear whether these methods can be successfully applied to multivariate VaR modelling.

${ }^{5}$ Filtered historical simulation is an exception. Although it uses recent data to capture time-varying volatility, it can simulate from a long time series of historical shocks, thus allowing it to do a better job of capturing event risk than the other methods listed in Table 1.

${ }^{6}$ If the portfolio in question consists of linear instruments, valuation is trivial. In general, portfolios contain non-linear instruments. One way to handle revaluation in this case is to produce a grid for each instrument, showing the change in its value as a function of the change in market risk factors. Many "front office" systems routinely produce such a grid for the value of options positions as a function of the underlying and its implied volatility. When grids are used, an accurate VaR can be computed via a grid Monte Carlo method. Pritsker (1997) demonstrates the use of a grid Monte Carlo method. 


\section{The Jump-VaR model}

The Jump-VaR model is based on the assumption that shocks to market risk factors follow some probability distribution in "ordinary" times (when there are no jumps). This distribution can have time-varying volatility. Any of the distributions that are commonly assumed in a VaR model (such as those listed in Table 1) would be a good candidate.

The Jump-VaR model combines a shock drawn from the "ordinary" distribution with a "jump" shock. Each day, the jump shock can produce a jump up, a jump down, or no jump. The possibility of a jump will enable the model to capture event risk. The "ordinary" and "jump" shocks are assumed to be independent of each other.

Section 3.1 gives a simple illustration of how the Jump-VaR model incorporates price jumps to produce "fatter" tails. Sections 3.2 and 3.3 give a rigorous and complete description of the Jump-VaR model.

\subsection{A simple illustration}

In this section of the paper, I show how the Jump-VaR model would represent the distribution of the return on a single large-cap stock on January $4,1999 .^{7}$ The model assumes

$$
\text { stock return }=\text { "ordinary" shock }+ \text { jump shock }
$$

where for this illustration the probability distribution of the jump shock is as follows:

$$
\text { jump shock }=\left\{\begin{array}{cc}
-7.5 \text { percent } & \text { with probability } .01 \\
+7.5 \text { percent } & \text { with probability } .01 \\
0 & \text { with probability } .98
\end{array}\right.
$$

I will model the distribution of the "ordinary" shock first with the HS distribution from Table 1. Then I will repeat the illustration using the VCV distribution. ${ }^{8}$

Figure 1 shows the probability density of the stock returns for the HS model. Comparing Panel A of the Figure, with no jumps, and Panel B, with jumps, it is clear that adding jumps puts more of the weight of the distribution into the tails, where the jumps occur. For the VCV model, the probability density with and without jumps is shown in Figure 2. Comparing them, the "jump" distribution clearly has fatter tails. In both cases (HS and VCV), adding event risk to the model puts more weight out in the tails of the distribution where jumps occur. As discussed above, a lack of "fat tails" is the reason why many VaR models do not accurately capture event risk.

Another way to illustrate the effect of adding event risk to the model is to look at the VaR estimates produced by the model, with and without jumps. Table 2 shows the one-day VaR estimates for a long position in the stock. VaR is computed for four different models

\footnotetext{
${ }^{7}$ The stock is General Electric.

${ }^{8}$ In these illustrations, HS uses 250 days of historical returns. VCV uses a multivariate normal distribution with decay factor 0.94 .
} 
(HS and VCV, with and without jumps), at both the 95 and 99 percent confidence levels. In all cases, adding jumps increases the VaR estimate. Adding jumps has only a small effect on the 95 percent VaR. For the 99 percent VaR, adding jumps increases the VaR estimates by a great deal. ${ }^{9}$ It is not surprising that "fattening" the tails of the distribution by adding jumps has more impact on the estimated VaR farther out in the tails of the distribution.

The next two subsections present a detailed development of the model. A reader whose main interest is in seeing an empirical implementation of the model can skip to section 4 on page 7 .

\subsection{Univariate model}

Suppose that the market risk factor $X$ can be written as the sum of a "ordinary" component $X_{0}$ and a "jump" component $X_{J}$ :

$$
X=X_{0}+X_{J}
$$

where $X_{0}$ has some distribution $F_{X_{0}}(x)$ (to be specified later), the jump component $X_{J}$ has a trinomial distribution

$$
X_{J}= \begin{cases}0 & \text { with probability } 1-p-q \\ -D & \text { with probability } p \\ U & \text { with probability } q,\end{cases}
$$

and $X_{0}$ and $X_{J}$ are independent. ${ }^{10}$ Given these assumptions, $X$ is equivalent to a mixture of three distributions:

$$
X=(1-p-q) X_{0}+p X_{D}+q X_{U}
$$

where $X_{D}$ has the same distribution as $X_{0}$ with the mean shifted by $-D, X_{U}$ has the same distribution as $X_{0}$ with the mean shifted by $U$, and $X_{0}, X_{D}$, and $X_{U}$ are all independent of each other.

This distribution can obviously capture jumps, with the jump component $X_{J}$. It can capture time-varying volatility by choosing the "ordinary" distribution $X_{0}$ appropriately. Note that, even if the volatility of $X_{0}$ is allowed to change over time, the jump parameters $(p$, $q, D, U)$ will be held constant. The infrequency of jumps makes it difficult enough to estimate an unconditional jump model; a conditional jump model would be even more difficult. The model can also capture skewness if $D \neq U$ or $p \neq q$. This model is similar to models in the finance literature, such as jump diffusion models, that describe the statistical distribution of stock returns as the sum of an "ordinary" component and a "jump" component. ${ }^{11}$

\footnotetext{
${ }^{9}$ Of course, in a simple illustration like this one, there is no way to tell which of the VaR estimates in Table 2 is the most accurate.

${ }^{10} X_{J}$ can also be interpreted as arising from the conditional distribution of three independent Poisson random variables (for no jump, jump down, and jump up) with expected values $1-p-q, p$, and $q$, conditional on the sum of the three equalling one. See Johnson, Kotz and Balakrishnan (1997, p. 32).

${ }^{11}$ For example, see Press (1967) and Ball and Torous (1983).
} 
To use this distribution in a VaR model, we either need to be able to draw random numbers from the distribution $F_{X}$ to be used in a Monte Carlo method or to evaluate the distribution function $F_{X}$ analytically. The distribution function of $X_{0}$ has already been specified as $F_{X_{0}}(x)$. Write this distribution function as $F_{X_{0}}(x ; \mu, \psi)$ where $\mu$ is the distribution's mean and the distribution's remaining parameters are stacked in the vector $\psi$. From (2), we know the distribution function of $X, F_{X}(x)$, can be written as

$$
F_{X}(x)=(1-p-q) F_{X_{0}}(x ; \mu, \psi)+p F_{X_{0}}(x ; \mu-D, \psi)+q F_{X_{0}}(x ; \mu+U, \psi) .
$$

As long as there is an analytic formula for $F_{X_{0}}$, there will be an analytic formula for $F_{X}$, which would make it easy to compute and invert $F_{X}$ as needed to compute an analytic VaR. It would be easy to draw random numbers from the distribution $F_{X}$ to compute Monte Carlo VaR using (1) directly.

\subsection{Multivariate model}

In practice, VaR is nearly always measured in a multivariate context. In this subsection, I present the multivariate generalization of the model given above. I will now use $X$ to refer to an $n \times 1$ vector of market risk factors:

$$
X=\left(X_{1} X_{2} \ldots X_{n-1} X_{n}\right)^{\prime}
$$

The issue of correlation is crucial to multivariate modelling of market risk factors. In a multivariate context, we care about the risk of correlated jumps. For measuring market risk, we care about correlated jumps over a short time horizon (e.g., one day or ten days). The characteristics of the portfolio will dictate the correlation assumption that is appropriate for that portfolio. Because correlation is crucial, it will be important to validate whatever correlation assumption is made.

One reasonable way of modelling the correlation of event risk is to assume that, for market risk factors related to the same event, the jumps are correlated. Otherwise, jumps in market risk factors are assumed to be independent. For example, in a convertible bond portfolio, the credit spread, equity price, and implied volatility for a single firm could be assumed to have correlated jumps. Risk arbitrage would be another portfolio where correlated jumps would be an issue. ${ }^{12}$

In the example I present in section 4 below, I measure the risk of a diversified portfolio of equities. For equities, it appears reasonable as a first approximation to model events as independent across market risk factors. ${ }^{13}$ With this "zero correlation" assumption, the fundamental model for $X$ becomes

$$
X=X_{0}+\sum_{i=1}^{n} X_{J_{i}}
$$

\footnotetext{
${ }^{12}$ For one treatment of correlated jumps, see Duffie and Singleton (1999).

${ }^{13} \mathrm{Keep}$ in mind that market-wide "events" should be captured in $X_{0}$. In section $4.4 \mathrm{I}$ present evidence on the assumption of independent jumps.
} 
where $X_{0}$ has a distribution function $F_{X_{0}}$, there is a jump component associated with each risk factor

$$
X_{J_{i}}= \begin{cases}0 & \text { with probability } 1-p_{i}-q_{i} \\ -D_{i} & \text { with probability } p_{i} \\ U_{i} & \text { with probability } q_{i}\end{cases}
$$

and the $X_{J_{i}}$ are independent of $X_{0}$ and $X_{J_{k}}$ for $i \neq k$.

As in the univariate case, $X$ can be viewed as a mixture. However, in the multivariate case, there are $3^{n}$ components ( 3 possible outcomes for each of $n$ independent trinomial random variables). This makes the mixture version of the multivariate model less appealing. ${ }^{14}$ Because of this, it will almost always be easier to work directly with (4), rather than a mixture version.

However, there could be some circumstances where a mixture version of (4) is useful. If a parametric model is being used for $X_{0}$, it could be desirable to estimate the parameters of that model in a mixture model framework. (I do this in section 4 for a VCV model.) Some simplification is needed to reduce the number of components to a tractable number. I suggest dropping the mixture components that correspond to two or more simultaneous jumps, which according to the model are quite unlikely to occur, and reassigning their probability mass to the $2 n$ mixture components corresponding to a single jump. This will create a mixture with $2 n+1$ components ( $2 n$ single-jump components and one no-jump component).

This model can be written as

$$
X=\left(1-\sum_{i=1}^{n} \hat{p}_{i}-\sum_{i=1}^{n} \hat{q}_{i}\right) X_{0}+\sum_{i=1}^{n} \hat{p}_{i} X_{D_{i}}+\sum_{i=1}^{n} \hat{q}_{i} X_{U_{i}}
$$

where the modified probabilities of the single jump states, $\hat{p}_{i}$ and $\hat{q}_{i}$, are defined as

$$
\hat{p}_{i}=p_{i}\left(\prod_{j \neq i}\left(1-p_{j}-q_{j}\right)\right)\left(\frac{1-\prod_{j=1}^{n}\left(1-p_{j}-q_{j}\right)}{\sum_{j=1}^{n}\left(p_{j}+q_{j}\right) \prod_{k \neq j}\left(1-p_{k}-q_{k}\right)}\right)
$$

and

$$
\hat{q}_{i}=\hat{p}_{i}\left(\frac{q_{i}}{p_{i}}\right)
$$

Each modified probability is set by taking the unmodified probability (in (6), this probability is $\left.p_{i}\left(\prod_{j \neq i}\left(1-p_{j}-q_{j}\right)\right)\right)$ and inflating it by a factor (the third term in $\left.(6)\right)$ that ensures the $2 n$ modified single-jump probabilities and the no-jump probability sum to one.

As in the univariate case, $X_{D_{i}}$ has the same distribution as $X_{0}$ with the mean of $X_{i}$ shifted by $-D_{i}, X_{U_{i}}$ has the same distribution as $X_{0}$ with the mean of $X_{i}$ shifted by $U_{i}$, and $X_{0}$, $X_{D_{i}}$, and $X_{U_{i}}$ are independent of each other, for all $i$. The magnitude of the misspecification

\footnotetext{
${ }^{14}$ For example, if $n=30,3^{n} \approx 306$ trillion.
} 
error introduced by ignoring the $3^{n}-(2 n+1)$ outcomes with two or more jumps will be larger, the greater are $n, p_{i}$ and $q_{i}$.

Again, as in the univariate case, the distribution function of $X$ will be a function of the distribution function of $X_{0}$. In the special case of linear exposures and $X_{0}$ distributed multivariate normal, VaR can be computed analytically from (5). For other, more realistic cases, analytic methods are unlikely to be feasible; Monte Carlo is the best choice of method in these cases. Random draws from the distribution of $X$ can be made easily using (4) as long as it is possible to make random draws from the distribution of $X_{0}$. The portfolio's value for each draw of $X$ can be computed directly, for linear instruments, or using grids for nonlinear instruments. Note that a large number of draws will be needed to ensure that the jumps are adequately captured.

\subsection{Choosing a model for "ordinary" times}

What distribution should be chosen to capture "ordinary" fluctuations? One strength of the Jump-VaR model is that it can incorporate any model for ordinary times. The models listed in Table 1 are common in the market and can capture time-varying volatility. The methodology I present would work with any of them.

\section{Estimating Jump-VaR for a portfolio of equities}

In this section, I use the Jump-VaR methodology to measure VaR of a portfolio of equities. The purpose of this empirical example is twofold: to help the reader understand how the Jump-VaR methodology works by walking through a detailed example, and to see whether including event risk affects the VaR estimates a lot, a little, or not at all.

The first step in using the Jump-VaR model is to estimate the model's parameters. The parameters include both the jump parameters $(p, q, D, U)$ and any parameters of the model assumed for "ordinary" times. I will estimate the parameters using data on a large number of equities. By using a large number of equities, rather than a specific portfolio, the estimated parameters should be able to be used on any portfolio of equities. In a realworld risk management environment, the estimation step would be done infrequently. The second step is to choose a specific portfolio of equities and estimate its VaR. In a real-world situation, this step would be done frequently (daily in most banks).

To show how the Jump-VaR model can be used with any model for "ordinary" times, I will run the empirical example twice, once using historical simulation (HS) and once using a variance-covariance model (VCV) as the distribution in "ordinary" times.

\subsection{Estimating parameters}

There are 4 jump parameters for each equity in the portfolio, or $4 n$ jump parameters in all $\left(p_{1}, q_{1}, D_{1}, U_{1}, \ldots, p_{n}, q_{n}, D_{n}, U_{n}\right)$. Rather than try to estimate different jump parameters for each equity, I choose to group equities into "buckets" with similar jump characteristics. 
Each equity's jump parameters will be those estimated for a "bucket" of equities with similar characteristics. This provides a much larger data sample for estimating the jump parameters and should result in more accurate estimated jump parameters, as long as the "buckets" are chosen appropriately.

Firm size is known to be strongly correlated with a stock's volatility. Therefore, I will use market capitalization as the characteristic determining an equity's jump parameters. I will sort firms into three size buckets - large-cap, mid-cap, and small-cap - and estimate a different set of jump parameters $(p, q, D, U)$ for each group. I also investigated using industry as a jump characteristic, but I did not find much evidence that jumps varied by industry.

What data should be used to estimate jumps? Obviously, a lot of data is needed to estimate the probability and magnitude of jumps, because jumps are infrequent. ${ }^{15}$ Fortunately, data on historical equity returns is easy to obtain. ${ }^{16}$ I used daily returns from the CRSP database over 1980-99. To begin, I divided the firms in each year into three groups based on their market capitalization. ${ }^{17}$ To keep the dataset size manageable, I randomly chose approximately 4,000 firm-years (approximately 1 million daily returns) from each size group to work with.

How the estimation step is done will depend on which model is being assumed for "ordinary" times. There is an important reason why: the jump parameters $(p, q, D, U)$ cannot be estimated in isolation from the model for "ordinary" times, or double-counting will result. Double-counting, in this context, means allowing the same large price jumps to affect both the jump parameters and the distribution in "ordinary" times. Double-counting would inflate the tails of the modelled distribution and result in an over-estimate of VaR.

\subsubsection{Estimating parameters for historical simulation}

When HS is the model assumed for "ordinary" times, the only parameters to be estimated are the jump parameters. HS is a "nonparametric" technique; it has no parameters to estimate. The only decision is how many days of historical data to use. In my example, I use 250 days (1 year).

It is worth noting at this point that a distribution modelled by $\mathbf{H S}$ will not necessarily under-estimate the probability of large price jumps. If large price jumps are present in the data used for HS, they will be present in the modelled distribution as well. The problem in practice is that $\mathbf{H S}$ is typically done with too short a sample of historical data to accurately estimate the probability of jumps. For example, if the jump probability were 0.004, an HS VaR model that uses 250 days would only be expected to record one jump in its data sample. It would be difficult to accurately estimate the contribution of event risk to VaR using HS

\footnotetext{
${ }^{15}$ Because I assume uncorrelated jumps for the equities in my sample portfolio, I do not have to worry about estimating correlated jumps, which could require even more data.

${ }^{16}$ For other portfolios, such as high-yield debt, there may not be enough historical data available to accurately estimate a jump model.

${ }^{17}$ In 1999, the lower cutoffs of the three groups were set at $\$ 50$ million, $\$ 1$ billion, and $\$ 12$ billion. Cutoffs for earlier years were set by deflating the 1999 cutoffs by changes in the S\&P 500 index.
} 
alone when the expected number of jumps in the data sample is only one. The Jump-VaR model combines HS with a model of event risk, which should make it easier to accurately measure VaR. If HS VaR were computed using a large enough set of historical data, there would be no need to separately account for event risk.

I will estimate the jump probabilities in each market capitalization bucket as follows. First, divide all equity returns into three groups: down jumps, up jumps, and no jumps. I arbitrarily set the cutoff between the groups at $\pm 4 \sigma$, where $\sigma$ refers to the sample standard deviation of returns in this particular bucket of equities. In effect, I assume that returns in excess of $\pm 4 \sigma$ cannot come from "ordinary" times and must have a jump component. Second, estimate the jump probabilities for this bucket as the percentage of returns falling in the "down jump" and "up jump" groups. Third, estimate the jump sizes for this bucket as the mean return in the "down jump" and "up jump" groups.

The estimated jump parameters for large-cap, mid-cap, and small-cap equities are shown in Table 3. The model picks up two well-known features of the distribution of returns on individual equities: positive skewness and an inverse relationship between firm size and the magnitude of jumps (larger firms have smaller jumps). Interestingly, the model suggests that the magnitude of a jump varies more strongly than the probability of a jump as firm size changes. The table also shows the average waiting time until the next jump. Based on these waiting times, I conclude that the average stock experiences a down jump every 1.5 to 2 years, and an up jump every 0.8 to 1.5 years.

The jump parameters are estimated from data pooled over 1980-99 under the assumption that the parameters are stable over time. This assumption should be verified. To do this, I dropped all observations from 1980-89 and re-estimated the model on data from the 1990s only. The jump parameters did not change appreciably. ${ }^{18}$

Simply plugging these jump parameters into the Jump-VaR methodology under HS would result in double-counting. I adopt the following ad hoc approach, which will reduce the extent of double-counting. For each equity in the portfolio, calculate the jump probabilities, both up and down, in the one year of historical data used for estimating VaR in "ordinary" times (the HS data sample). ${ }^{19}$ If the equity's "one-year jump probability" is less than the twenty-year jump probability for the appropriate bucket of equities, the $\mathbf{H S}$ data sample will not produce a "thick" enough tail for the probability distribution of the equity's returns. For these equities, "thicken" the tail by setting the equity's jump probability equal to the jump probability for the appropriate bucket in Table 3 and zero out this equity's jumps in the HS data sample used for "ordinary" times to avoid double-counting.

On the other hand, if the equity's one-year jump probability is greater than the twentyyear jump probability, the tail of the distribution is already "thick." In this case, set the equity's jump probability to zero and allow the jumps in the HS data sample to remain. By not adding jumps for such equities, double-counting can be avoided. The resulting VaR estimate will be conservative since equities with a low incidence of jumps within the $\mathbf{H S}$ data

\footnotetext{
${ }^{18}$ The jump probabilities fell slightly and the jump sizes rose. The expected jump (probability times size) rose by about 10 percent.

${ }^{19}$ Use the same cutoffs as described above to calculate jump probabilities.
} 
sample have their tails "thickened," while equities with a high incidence of jumps within the HS data sample do not have their tails "thinned."

\subsubsection{Estimating parameters for a VCV model}

The leading statistical technique for estimating a time-varying variance-covariance matrix is multivariate GARCH modelling. There are many different multivariate GARCH models, ranging from simple models like RiskMetrics ${ }^{\mathrm{TM}}$ to very complex models. ${ }^{20}$ For estimating VaR, one will typically need to work with a large number of market risk factors. This suggests a need to stick with the simplest possible GARCH models. In order to allow volatility to be mean reverting, I prefer to use the scalar GARCH model.

In a scalar GARCH model, each variance and covariance is modelled as following a GARCH $(1,1)$ process depending on its own past squared returns and cross-products of returns, respectively. The parameters of the GARCH process are restricted to be the same for each variance and covariance. ${ }^{21}$ The variance-covariance matrix, $H_{t}$, evolves according to

$$
H_{t}=S(1-\alpha-\beta)+\alpha \epsilon_{t-1} \epsilon_{t-1}^{\prime}+\beta H_{t-1}
$$

where $S$ is the long-run variance-covariance matrix, assumed to equal the sample average variance-covariance matrix. There are two parameters to be estimated, $\alpha$ and $\beta$. $\alpha$ captures the sensitivity of each variance and covariance to yesterday's return, while $\beta$ captures persistence.

I choose to estimate a scalar GARCH model with normally-distributed errors, rather than errors drawn from a fat-tailed distribution. I do this for two reasons. First, I am already "fattening" the tails of the distribution by adding jumps. The most common multivariate Student $t$ distribution imposes the same degree of "fat tails" on each series. The Jump-VaR model allows each series' "fat tails" to vary according to the probability of a jump in that series. So the Jump-VaR model is more flexible at capturing jumps when the frequency of jumps varies across equities. Second, it is still common for banks to use VaR models that assume a normal distribution, so this model is a useful benchmark.

The scalar GARCH model to be estimated is

$$
\begin{aligned}
& X_{t}=H_{t}^{1 / 2} \nu_{t}+X_{J, t} \\
& H_{t}=S(1-\alpha-\beta)+\alpha X_{t-1} X_{t-1}^{\prime}+\beta H_{t-1}
\end{aligned}
$$

where $X_{t}$ is a $30 \times 1$ vector of daily equity returns, $H_{t}$ is the variance-covariance matrix, $\nu_{t}$ is a multivariate standard normal random vector, and the jump term $X_{J, t}$ is the second term in (4). There are two parameters to be estimated, $\alpha$ and $\beta$, in addition to the jump parameters $p, q, D$, and $U$.

I estimate this model eight times, using daily returns on eight different sets of thirty equities each over 1994-98. Because I want to identify the GARCH parameters that are

\footnotetext{
${ }^{20}$ Engle and Mezrich (1996) survey multivariate GARCH models.

${ }^{21}$ I adopt the suggestion of Engle and Mezrich (1996) to constrain the long-run variances and covariances to be equal to their sample analogues.
} 
applicable across the entire asset class of equities, not those that apply to a particular equity portfolio, I choose to estimate the model on eight different sets of thirty equities and take the average values of the parameters as "the" parameters to be used when estimating Valueat-Risk. $^{22}$

The GARCH estimation must be done at the same time the jump parameters are estimated, to avoid double-counting. However, standard numerical optimization algorithms have a hard time estimating the jump parameters. I proceed to estimate the scalar GARCH model with jumps as follows. I begin by setting the jump parameters equal to those estimated for the HS model (shown in Table 3). The jump parameters vary with market capitalization as in Table 3. Next, I allow the jump probabilities to differ from those in Table 3 by a scaling parameter $\lambda$. That is, for each equity $i$,

$$
p_{i}=\lambda \hat{p}_{i} \quad q_{i}=\lambda \hat{q}_{i}
$$

where $\hat{p}_{i}$ and $\hat{q}_{i}$ are the $p$ and $q$ from Table 3 appropriate for the firm's market capitalization. The scaling parameter $\lambda$ is constrained to be the same for all equities. The jump sizes $D$ and $U$ are not changed from those in Table 3. This technique allows the jump probabilities to be estimated at the same time as the GARCH parameters to avoid double-counting. The estimation is done via maximum likelihood, and the likelihood is computed using the mixture model (5). ${ }^{23}$

I estimated the scalar GARCH model with and without jumps. Results are given in Table 4. I focus on the mean parameter estimates across the eight estimations, since these are the parameters that I will use in the VCV VaR estimation to follow. Looking at the GARCH parameters $\alpha$ and $\beta$, they indicate that shocks to volatility are persistent ( $\beta$ is close to one), but volatility is not too sensitive to shocks to equity returns ( $\alpha$ is close to zero). The $\alpha$ and $\beta$ estimated with and without jumps are not that different. The mean estimate of $\lambda$ is 0.67, implying that jump probabilities are two-thirds as large as those estimated in Table 3 for the HS model. The mean log-likelihood increases moving from the no-jump model to the jump model, indicating that the jump model fits the data better (as we would expect).

\subsection{Estimating VaR}

All the work has been done to conduct a VaR estimation for a portfolio of equities. I will estimate VaR using both the HS and VCV models, with and without jumps, using 5,000 Monte Carlo draws. I will work with two different portfolios. The "long-short portfolio" has equally-sized positions in 30 equities: 15 long positions and 15 short positions. This portfolio is hedged against general market moves, so all its market risk is specific risk. (Recall that specific risk includes both idiosyncratic risk and jump risk.) The "long portfolio" has a long

\footnotetext{
${ }^{22}$ Each set of 30 equities has 10 each of large-cap, mid-cap, and small-cap stocks, as do the sample portfolios used in section 4.2 below.

${ }^{23}$ The estimation was done in Gauss using the Constrained Maximum Likelihood module. The constraints on the model are $\alpha>0, \beta>0, \alpha+\beta \leq 1$. In the estimation, $H_{0}$ (the initial estimate of the variancecovariance matrix) was set equal to the sample variance-covariance matrix. The source code for the estimation is available on request.
} 
position of equal value in all 30 equities; specific risk is 56 percent of total market risk for this portfolio. ${ }^{24}$ Both portfolios have 10 large-cap, 10 mid-cap, and 10 small-cap equities. Since the jump and GARCH parameters were estimated over 1994-98, I will estimate VaR for the 252 trading days in 1999 to test the models' out-of-sample performance.

\subsubsection{VaR of the long-short portfolio}

The 99 percent one-day VaR estimates from the Jump-VaR model and the standard (nojump) VaR model estimated on the long-short portfolio are shown in Figure 3, along with the actual profit or loss $(\mathrm{P} / \mathrm{L})$ on the portfolio for each day. Panel A and Panel $\mathrm{B}$ of the figure show the VaR estimates using the HS and VCV models, respectively. It can be clearly seen how the Jump-VaR model results in an increase in VaR, relative to the no-jump model.

The estimated VaR results are summarized in Table 5. The first two columns of the table show the mean VaR over the 252 trading days in 1999, for both the left and right tails. The last two columns show the number of violations (days that $\mathrm{P} / \mathrm{L}$ exceeded VaR). For a 99 percent VaR over 252 days, the expected number of violations in each tail is 2.52 . VaR estimates are shown for both the HS and VCV models.

For each model, the first of the three rows in the table reflects the VaR estimates when no jumps are added. This is what a standard VaR model would produce. The second row adds jumps but does not correct for double-counting. The second row must be greater (in absolute value) than the first by construction, since jumps are being added to fatten the tails. The third row is the Jump-VaR model, which both adds jumps and corrects for double-counting. The third row must be less (in absolute value) than the second row by construction, since the double-counting adjustment thins the tails to avoid double-counting. The difference between the standard VaR model in the first row and the Jump-VaR model in the third row shows the full effect of incorporating jumps and correcting for double-counting.

Table 5 shows that incorporating event risk has a large effect on VaR for the long-short portfolio. The mean Jump-VaR is as much as 12 percent higher than the mean no-jump VaR. The number of violations is quite high for the no-jump VaR and much lower (and close to the expected number) for Jump-VaR. Still, neither the no-jump model or the Jump-VaR model would be rejected in a formal, statistical sense. ${ }^{25}$

\subsubsection{VaR of the long portfolio}

The VaR estimates and P/L for the long portfolio are shown in Figure 4, which follows the same format as Figure 3. Comparing the two figures, it is clear that the differences between the no-jump VaR and the Jump-VaR are much smaller for the long portfolio than for the long-short portfolio.

\footnotetext{
${ }^{24}$ The fraction of total market risk that is specific risk is estimated as $\left(1-R^{2}\right)$ from a regression of the long portfolio's one-day P\&L on the one-day return of the S\&P 500 index. The regression is run over the 252 trading days in 1999.

${ }^{25}$ For a sample of 252 days, the simple statistical test of a VaR model based on the number of violations (a binomial test) will reject the model when the number of violations is seven or greater. See Kupiec (1996, Table 5).
} 
The 99 percent one-day VaR estimates on the long portfolio are summarized in Table 6, which follows the same format as Table 5. As was clear from the figures, for this portfolio, incorporating event risk makes only a small difference to the estimate of VaR. This illustrates the point that the importance of event risk to VaR can vary greatly according to the nature of the portfolio whose risk is being measured.

\subsubsection{Comparing no-jump VaR and Jump-VaR}

Tables 5 and 6 allow one to compare the mean VaR estimates for the 252 trading days in 1999. However, as is clear from Figures 3 and 4, on some of the days in 1999 the difference between the no-jump and Jump-VaR models was much larger than the average difference shown in the tables. If we are interested in the answer to the question "How bad can a no-jump VaR estimate be?", we should look at the maximum difference between the two models, not the average difference.

Table 7 shows both the mean and maximum difference in VaR between the no-jump model and the Jump-VaR model. Three points are obvious from the table. First, the maximum differences between the two models are much larger than the average differences. For the long-short portfolio, on at least one day in 1999, the Jump-VaR model produced a 44 percent larger right-tail VaR estimate than the no-jump model. Second, although the mean differences in VaR are similar for the HS and VCV models, the same cannot be said of the maximum differences in VaR. The HS model appears to be more erratic than the VCV model when looking at the maximum difference in VaR. ${ }^{26}$ Third, contrary to what Table 6 suggested, incorporating jumps does make a difference for the long portfolio, at least for the HS model. On one day in 1999, the Jump-VaR estimate for the long portfolio was 24-26 percent larger than the no-jump VaR estimate.

Another way to compare no-jump VaR and Jump-VaR is to determine what the coverage level of the no-jump VaR model would be, if the Jump-VaR model were true. The coverage level is defined as the probability of a day's $\mathrm{P} / \mathrm{L}$ exceeding VaR, assuming a particular probability distribution for $\mathrm{P} / \mathrm{L}$. For an $P$ percent $\mathrm{VaR}$ estimate based on the true distribution of $\mathrm{P} / \mathrm{L}$, the coverage level would be $1-P$ percent. I note that the coverage level and the rate of VaR violations, discussed above, both measure how much probability mass is present in the tail of the $\mathrm{P} / \mathrm{L}$ distribution in excess of $\mathrm{VaR}$. The coverage level uses a model-based probability distribution while the rate of $\mathrm{VaR}$ violations uses an empirical probability distribution based on actual $\mathrm{P} / \mathrm{L}$ data.

Table 8 presents the coverage level of the no-jump VaR model, under the assumption that the Jump-VaR model is true. While the Jump-VaR model is not literally true, it is likely to be closer to the truth than the no-jump model. In the case of the VCV model, the Jump-VaR model is closer to the truth than the no-jump model in the sense of having a higher log-likelihood (see Table 4). In the case of the HS model, the difference between the no-jump model and the Jump-VaR model will depend on whether the particular HS data sample is one where the rate of price jumps is close to its long-run value or not.

\footnotetext{
${ }^{26}$ The volatile nature of the HS VaR estimates is also evident in Figure 3. Pritsker (2001) discusses some of the drawbacks of the HS method.
} 
The results in Table 8 suggest that, for the long-short portfolio, when the no-jump VCV model aims at producing a 99 percent VaR, it actually produces a 98.4 percent VaR on average. On its worst day (in terms of coverage level), it produces a 97.8 percent VaR. For the HS model, the left-tail no-jump VaR appears to be too low, producing 98.6 percent coverage, while the right-tail no-jump VaR appears to produce accurate coverage. For the long portfolio, as noted above, there are only small differences on average between the nojump VaR and Jump-VaR.

\subsection{Estimating ten-day VaR}

The VaR estimates presented above were all for a one-day horizon. A ten-day horizon may also be of interest, since a ten-day horizon is the basis for the regulatory capital requirement for market risk. To estimate ten-day VaR, I again use 5,000 Monte Carlo draws. For each Monte Carlo draw, ten one-day returns are drawn and are then compounded to produce a single ten-day return. For the VCV model, the variance-covariance matrix used to draw the second (and subsequent) one-day returns for each Monte Carlo draw is updated in the usual way (following equation (8)) using the preceding one-day return(s) for that Monte Carlo draw.

The ten-day VaR estimates are shown for the long-short portfolio in Table 9 and for the long portfolio in Table 10. Since the importance of correcting for double-counting has already been demonstrated for the one-day VaR estimates, the Jump-VaR estimates in Table 9 and Table 10 are all corrected for double counting. ${ }^{27}$

Overall, the ten-day VaR estimates tell the same story as the one-day VaR estimates. Just as for the one-day VaR estimates, the mean ten-day VaR estimates for the long-short portfolio increase by up to 14 percent when event risk is taken into account. For the long portfolio, estimated VaR changes little when jumps are added. In two of the four cases dealing with the long portfolio in Table 10, VaR actually declines slightly when jumps are added. For the long portfolio, jumps appear to affect the right-tail VaR more than the lefttail VaR. This reflects the well-known positive skewness of returns on individual stocks. ${ }^{28}$

Tables 9 and 10 also show the number of VaR violations. The number of violations is computed for the 25 non-overlapping ten-day intervals during 1999. Since there are only 25 observations, the expected number of violations for a 99 percent VaR is 0.25 . Thus it is not surprising that there were, in fact, no violations during these 25 ten-day intervals.

\subsection{Robustness of the VaR estimates}

I have implemented the Jump-VaR model for equities assuming that jumps are independent across firms. If this assumption does not hold in actual data, the tails of the distribution of

\footnotetext{
${ }^{27}$ Under a normal distribution, the ratio of ten-day VaR to one-day VaR would equal $\sqrt{10}$, or 3.16 . Comparing Tables 5 and 6 to Tables 9 and 10, these ratios range from just below 3 for the left-tail VaRs to around 3.25 for the right-tail VaRs.

${ }^{28}$ See Duffee (1995) for evidence.
} 
portfolio value will not be "thick" enough, because the likelihood of simultaneous jumps is underestimated. As a result, VaR could be underestimated.

I take the following approach to check this assumption. For a randomly chosen group of stocks, I count the number of jumps per day for each day in 1998 and 1999. I compare this distribution with the distribution implied under the independent jump hypothesis. If these two distributions are different, the hypothesis of independent jumps can be rejected.

I create a sample of 90 stocks by choosing 30 stocks with complete daily return histories over 1998-99 at random from each size group (large cap, mid cap, small cap). For each stock, each day's return is coded as either a jump or no jump for each of the 504 days in 1998-99. The jump thresholds for each size group are set as in section 4.1.1 above. On each day, I count the number of stocks that jumped (either up or down). The columns of Table 11 under the "Actual" heading show the tabulation of these counts.

Let the random variable $J$ denote the number of jumps among $N$ stocks, and let $J_{i}$ be a Bernoulli random variable that takes the value one if there is a jump (up or down) in stock $i$, and zero if there is no jump. By definition,

$$
J=\sum_{i=1}^{N} J_{i} .
$$

Under the Jump-VaR model, the probability of a jump (up or down) is

$$
\operatorname{Pr}\left\{J_{i}=1\right\}=p_{i}+q_{i} .
$$

$J$ follows a multinomial distribution with possible outcomes $0,1,2, \ldots, N$. Because the probabilities of individual stock jumps are different (they depend on market capitalization) and $N$ is large, the probabilities of each outcome for $J$ (under the null hypothesis of independent jumps) are non-trivial to compute. I use the formulae from Percus and Percus (1985, p. 624) for $\operatorname{Pr}\{J=j\}$ for $j=0,1,2,3,4 .^{29}$ These theoretical probabilities are shown in Table 11 in the columns headed "Theoretical".

It is clear from Table 11 that the actual and theoretical distributions are different. The two distributions have roughly the same number of 1 -jump days, but the actual data has many more days with more than 1 jump. This pattern is what one would expect if individual firms' jumps were modelled correctly, but correlated jumps were underestimated in the theoretical distribution. Some of the disparity between the two distributions may be due to market-wide jumps that the Jump-VaR model is not designed to capture. However, market-wide jumps cannot be the only reason for the disparity, since they would mostly affect the " 5 or more jumps" category, while the disparity is evident in the 2,3 , and 4 jump categories also. The usual statistical tests of goodness-of-fit easily reject the hypothesis that the actual data follow the theoretical distribution assuming independent jumps. ${ }^{30}$

As a consequence, the VaR estimates for the Jump-VaR model presented above, which were in some cases quite a bit larger than the no-jump VaR estimates, could still be too

\footnotetext{
${ }^{29}$ An approximation to the distribution of $J$ could be computed using the Poisson approximation to the binomial distribution. This approximation would be very close as the jump probabilities are close to zero.

${ }^{30}$ Both Pearson's $\chi^{2}$ test and a Kolmogorov-Smirnov test easily reject at the 1 percent level.
} 
small. In order to understand how important correlated jumps in equities are for capturing event risk in VaR, additional research into modelling correlated jumps is needed.

\section{$5 \quad$ Jump -VaR and market risk capital}

Since bank supervisors use VaR as the basis for the regulatory capital charge for market risk, moving from a standard (no-jump) VaR model to a Jump-VaR model would affect a bank's regulatory capital. ${ }^{31}$ The market risk capital charge is equal to

$$
3 \times \text { VaR from market-wide shocks }+m \times \text { specific risk VaR }
$$

where $m$ is the specific risk multiplier, equal to 4 if the VaR model does not capture event risk and 3 if it does. When moving from a standard (no-jump) VaR model to a Jump-VaR model, regulatory capital could rise or fall, since specific risk VaR would increase and the specific risk multiplier $m$ would decline from 4 to 3 .

Whether regulatory capital would rise or fall in any particular case would depend on the magnitude of the increase in $\mathrm{VaR}$ and the fraction of total VaR attributed to specific risk. Let regulatory capital be denoted by $K$, total VaR by $V$, and the fraction of VaR stemming from specific risk as $f$. Let subscripts " $N J$ " and " $J$ " denote the no-jump VaR and Jump$\mathrm{VaR}$, respectively. Using this notation and the formula for the market risk capital charge given in the previous paragraph, the capital charge using the no-jump VaR model is

$$
K_{N J}=3(1-f) V_{N J}+4 f V_{N J}
$$

and the capital charge using the Jump-VaR model is

$$
K_{J}=3 V_{J}
$$

Combining (9) and (10), it can be shown that regulatory capital will fall $\left(K_{J}<K_{N J}\right)$ if and only if the percentage increase in $\operatorname{VaR}\left(\frac{V_{J}-V_{N J}}{V_{N J}}\right)$ is less than $f / 3 .^{32}$

For both of the example portfolios considered in this paper, regulatory capital for market risk would fall when moving from a standard (no-jump) VaR model to the Jump-VaR model. For the long-short portfolio, the percentage increase in VaR is never greater than 12 percent, while $f / 3$ is 33 percent (since $f=1$ ). For the long portfolio, the percentage increase in VaR is never greater than 4 percent, while $f / 3$ is 19 percent (since $f=0.56$ ). The conclusion that regulatory capital falls is, of course, specific to these two portfolios.

\footnotetext{
${ }^{31}$ Regulatory capital is based on the ten-day VaR.

${ }^{32}$ Using (9) and (10),

$$
\begin{aligned}
K_{J} & <K_{N J} \\
3 V_{J} & <3(1-f) V_{N J}+4 f V_{N J} \\
3\left(V_{J}-V_{N J}\right) & <f V_{N J} \\
\frac{V_{J}-V_{N J}}{V_{N J}} & <\frac{f}{3}
\end{aligned}
$$
}




\section{Conclusions}

The goal of this paper is to illustrate the issues involved in adding event risk to VaR. In considering the nature of event risk and how to incorporate it into a VaR model, I have made several observations:

- event risk, jump risk, and fat tails are different names for the same phenomenon;

- to accurately measure event risk, a model must accurately measure the fat tails that are present in real financial data;

- a long data sample is needed to accurately capture the fat tails in financial data;

- a model's distributional assumption must be able to accommodate fat tails.

I presented the Jump-VaR model as a demonstration of one way to deal with these issues. It combines a standard VaR model for "ordinary" times with a simple model of price jumps. The effect is to fatten the tails of the distribution of returns and thereby capture event risk. The jump component can be estimated on a long data sample, even if the standard VaR model is not.

Some issues arose in the implementation of the Jump-VaR model that would have to be addressed in any model of event risk:

- correlation of jumps is a crucial issue that will have to be addressed on a portfolio-byportfolio basis;

- jumps can be estimated for a "basket" of similar securities, rather than for an individual security, to increase the effective size of the data sample;

- double-counting of jumps (i.e., including jumps in the standard VaR model as well as in the jump component) should be avoided;

I ran the Jump-VaR model on two equity portfolios, using both a historical simulation model and a variance-coavriance model as the standard VaR model for "ordinary" times. The results illustrated that the Jump-VaR model does thicken the tails of the distribution to account for event risk, thereby increasing VaR. In some cases, the increase in VaR was substantial. As expected, the increase in VaR was greater when the portfolio's exposure to specific risk was greater. For regulatory capital purposes, the increase in VaR would have been outweighed by the decline in the specific risk multiplier from 4 to 3, reducing the regulatory capital charge for market risk. It should be stressed that this final conclusion is specific to these two portfolios and would not necessarily carry over to other portfolios. 


\section{References}

Ball, Clifford A. and Walter N. Torous, 1983. A simplified jump process for common stock returns. Journal of Financial and Quantitative Analysis 18:1 (March), 53-65.

Barone-Adesi, Giovanni, Kostas Giannopoulos, and Les Vosper, 1999. VaR without correlations for portfolios of derivative securities. Journal of Futures Markets 19:5 (August), $583-602$.

Bollerslev, Tim, 1987. A conditionally heteroskedastic time series model for speculative prices and rates of return. Review of Economics and Statistics 69:3 (August), 542-547.

Boudoukh, Jacob, Matthew Richardson, and Robert Whitelaw, 1998. The best of both worlds. Risk (May).

Duffee, Gregory R., 1995. Stock returns and volatility: A firm-level analysis. Journal of Financial Economics 37:3 (March), 399-420.

Duffie, Darrell and Kenneth Singleton, 1999. Simulating correlated defaults. Working paper, Stanford Graduate School of Business.

Engle, Robert and Joseph Mezrich, 1996. GARCH for groups. Risk (August), 36-40.

Johnson, Norman L., Samuel Kotz and N. Balakrishnan, 1997. Discrete Multivariate Distributions. John Wiley and Sons.

Jorion, Philippe, 2000. Value at Risk: The New Benchmark for Managing Financial Risk. Second Edition. McGraw-Hill.

J.P. Morgan/Reuters, 1996. RiskMetrics ${ }^{\mathrm{TM}}$ Technical Document. Fourth Edition. (can be downloaded from http://www.riskmetrics.com)

Kupiec, Paul H., 1996. Techniques for verifying the accuracy of risk measurement models. In Risk Measurement and Systemic Risk: Proceedings of a Joint Central Bank Research Conference. Federal Reserve Board.

Linsmeier, Thomas J. and Neil D. Pearson, 2000. Value at Risk. Financial Analysts Journal 56:2 (March/April), 47-67.

Percus, Ora E. and Jerome K. Percus, 1985. Probability bounds on the sum of independent nonidentically distributed binomial random variables. Siam Journal on Applied Mathematics 45:4 (August), 621-640.

Press, S. James, 1967. A compound events model for security prices. Journal of Business 40:3 (July), 317-335. 
Pritsker, Matthew, 1997. Evaluating Value at Risk methodologies: Accuracy versus computational time. Journal of Financial Services Research 12:2-3 (October-December), 201-242.

Pritsker, Matthew, 2001. The hidden dangers of historical simulation. Working paper, Federal Reserve Board (January). 
Figure 1. Probability density of the Return on a Single LARGe-CAP Stock on January 4, 1999: Estimated By Historical Simulation

A. No jumps

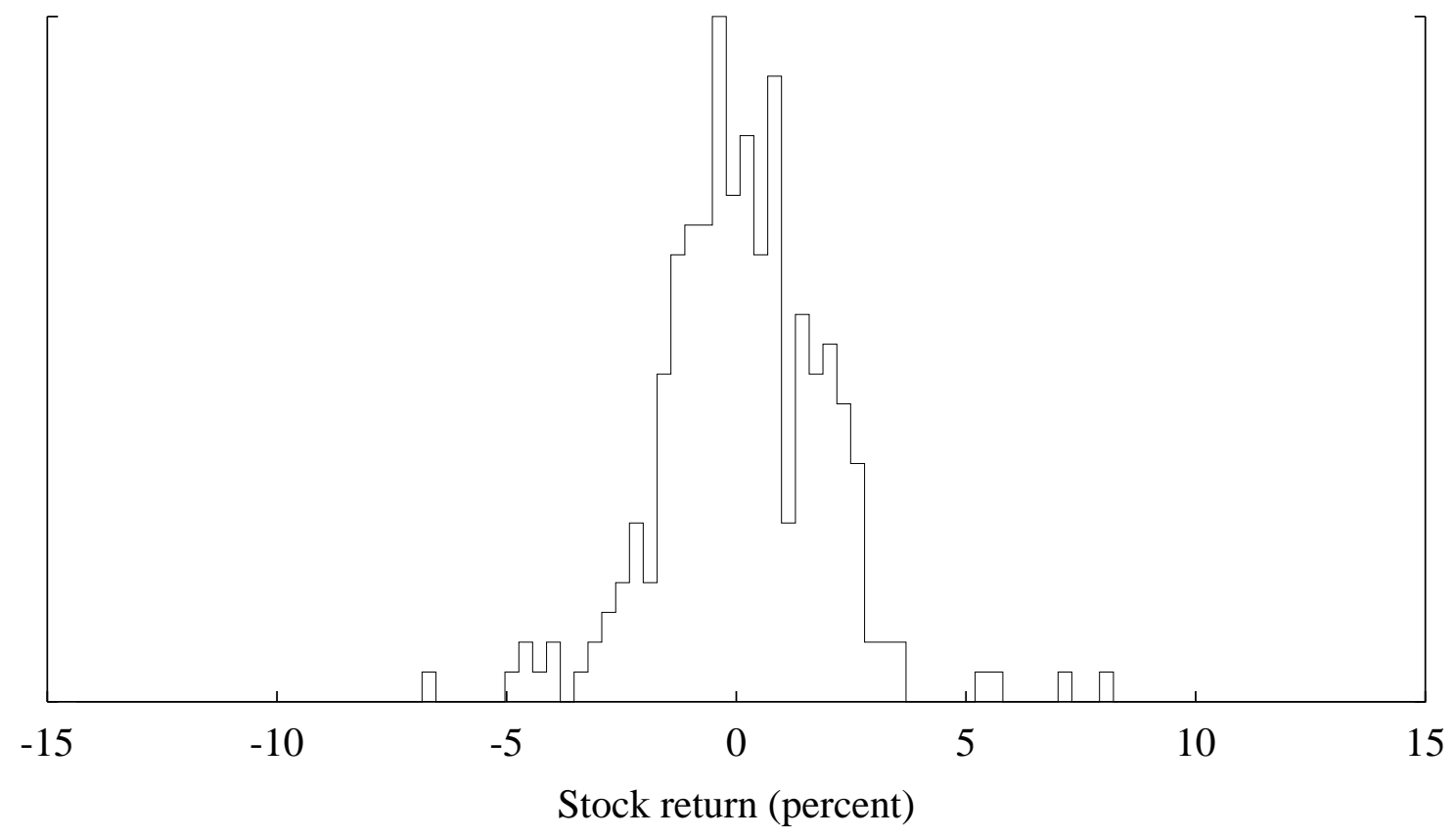

B. With jumps

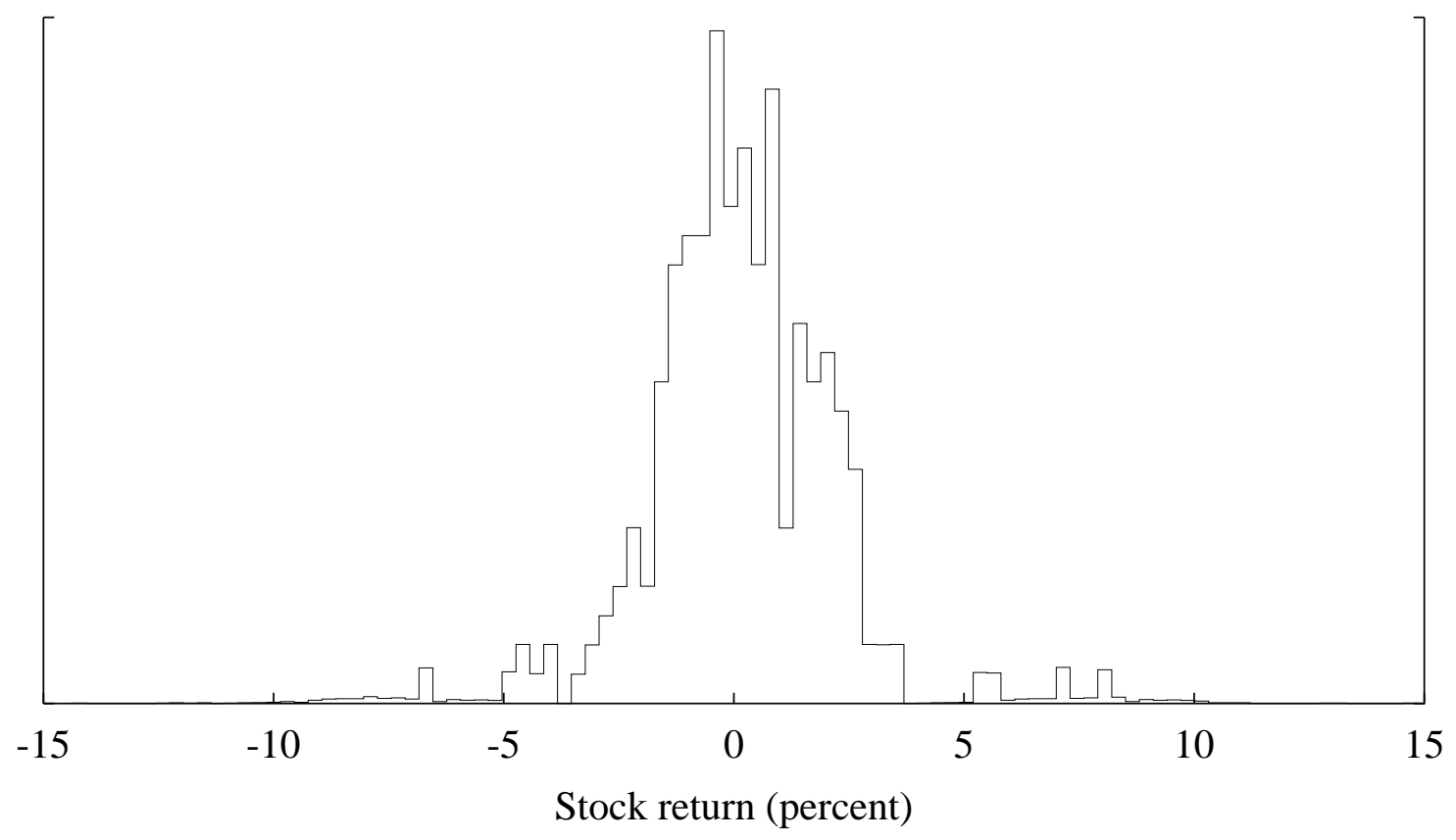


Figure 2. Probability Density of the Return on A Single LARGe-CAP STOCK ON JANUARY 4, 1999: Estimated WITH A VCV MODEL

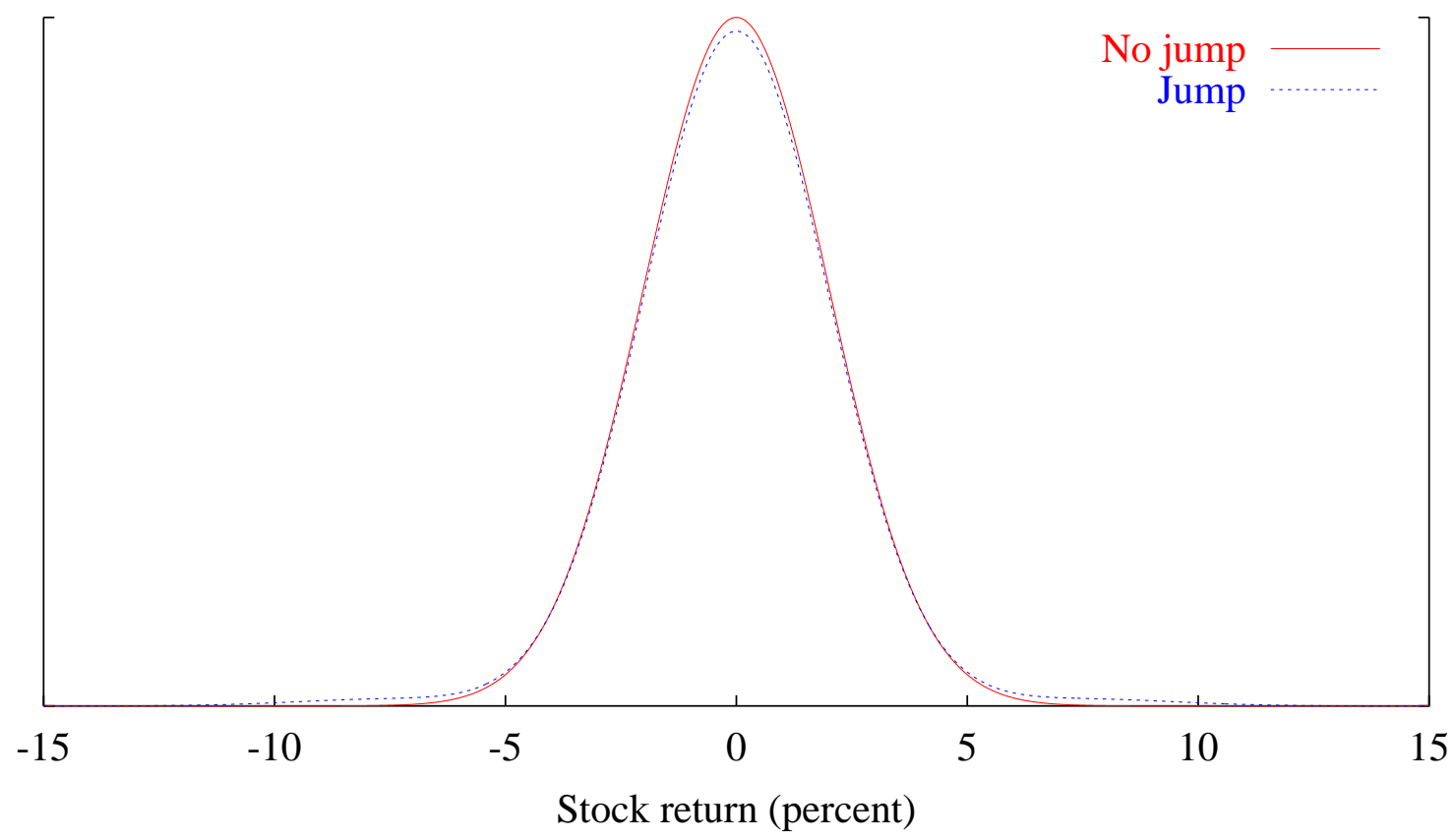


Figure 3. One-day 99 Percent VaR estimates And Realized P/L on the LONGSHORT PORTFOLIO

A. VaR estimated with the HS model

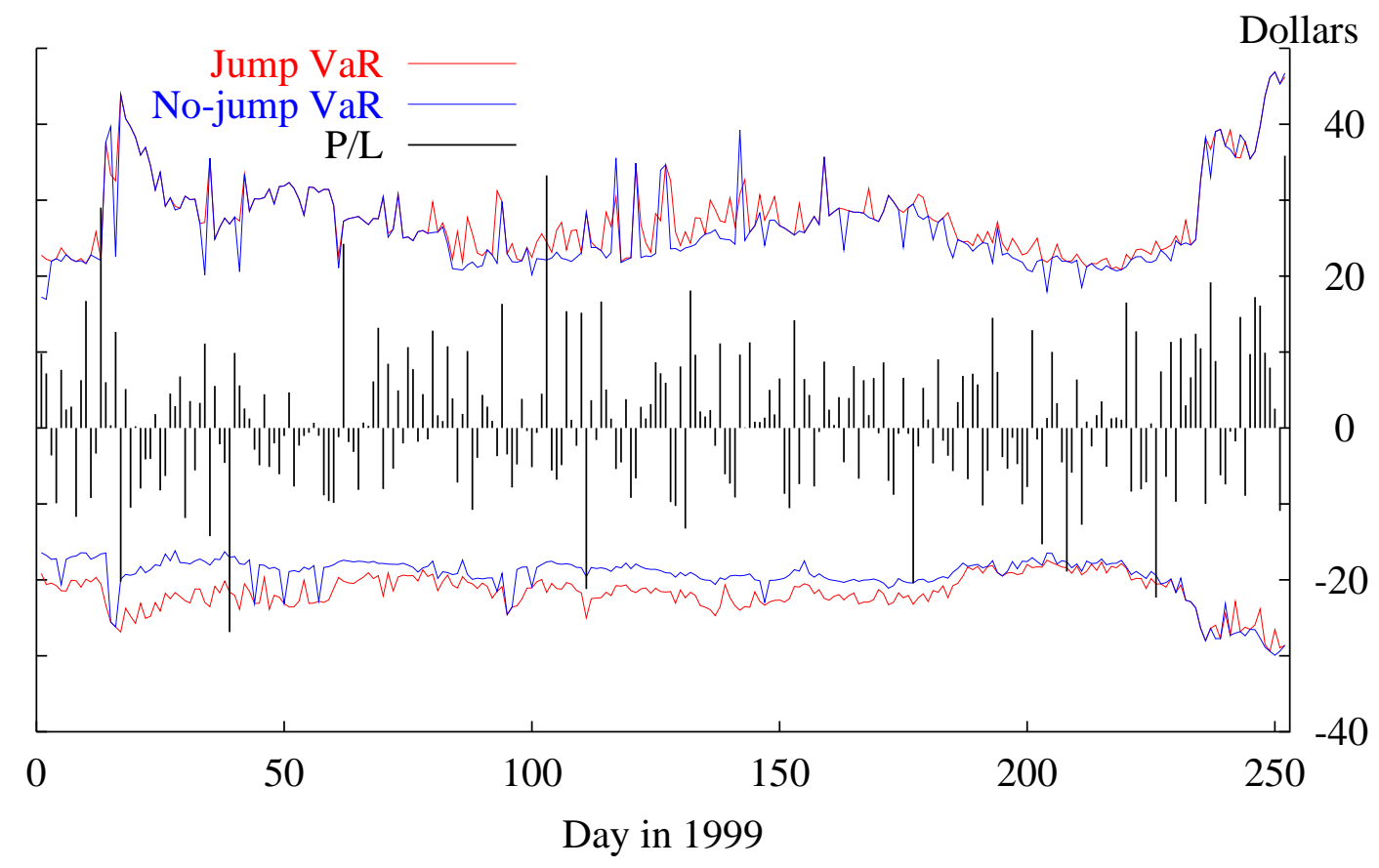

B. VaR estimated with the VCV model

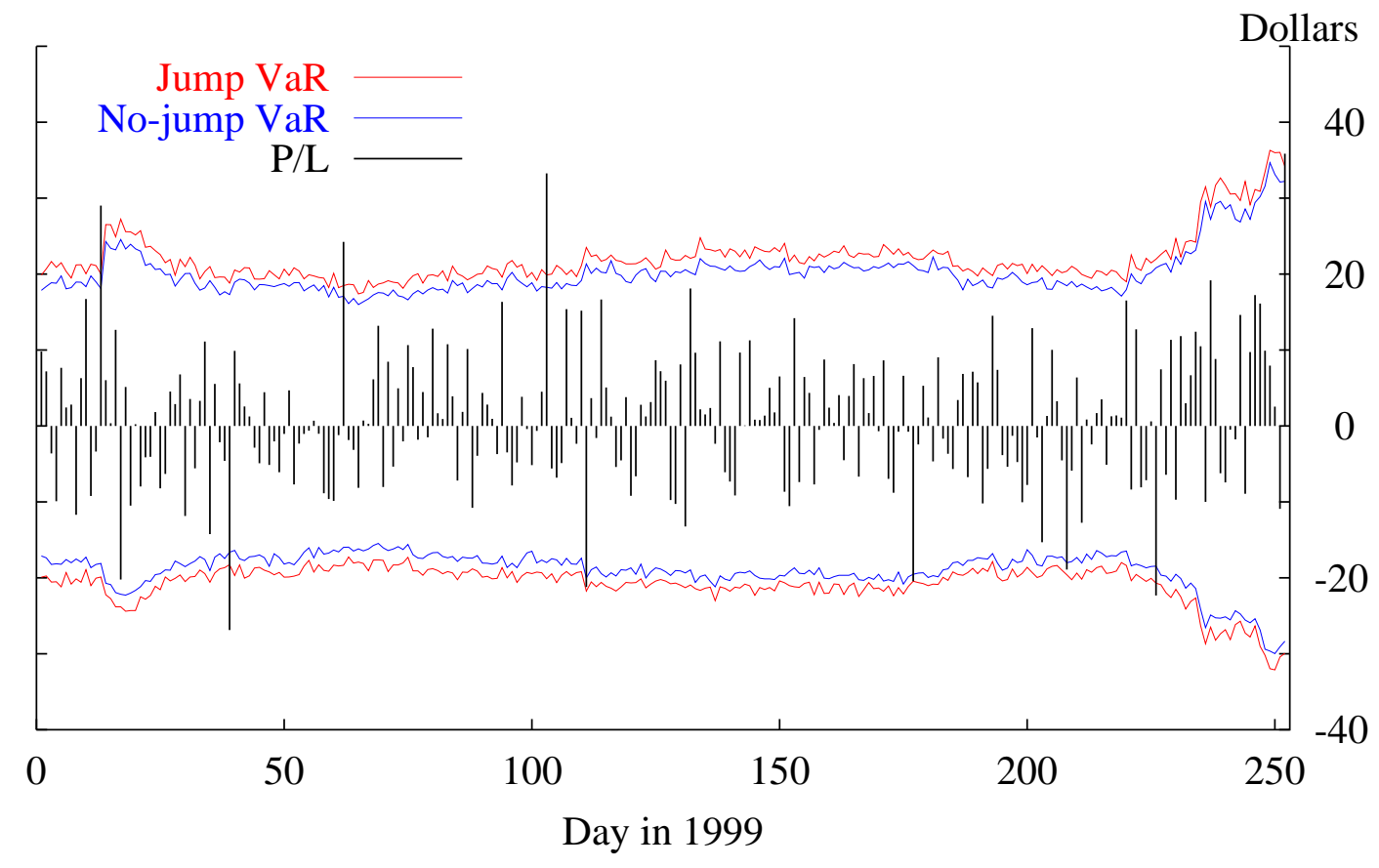


Figure 4. One-day 99 Percent VaR estimates and Realized P/L on the Long PORTFOLIO

A. VaR estimated with the HS model

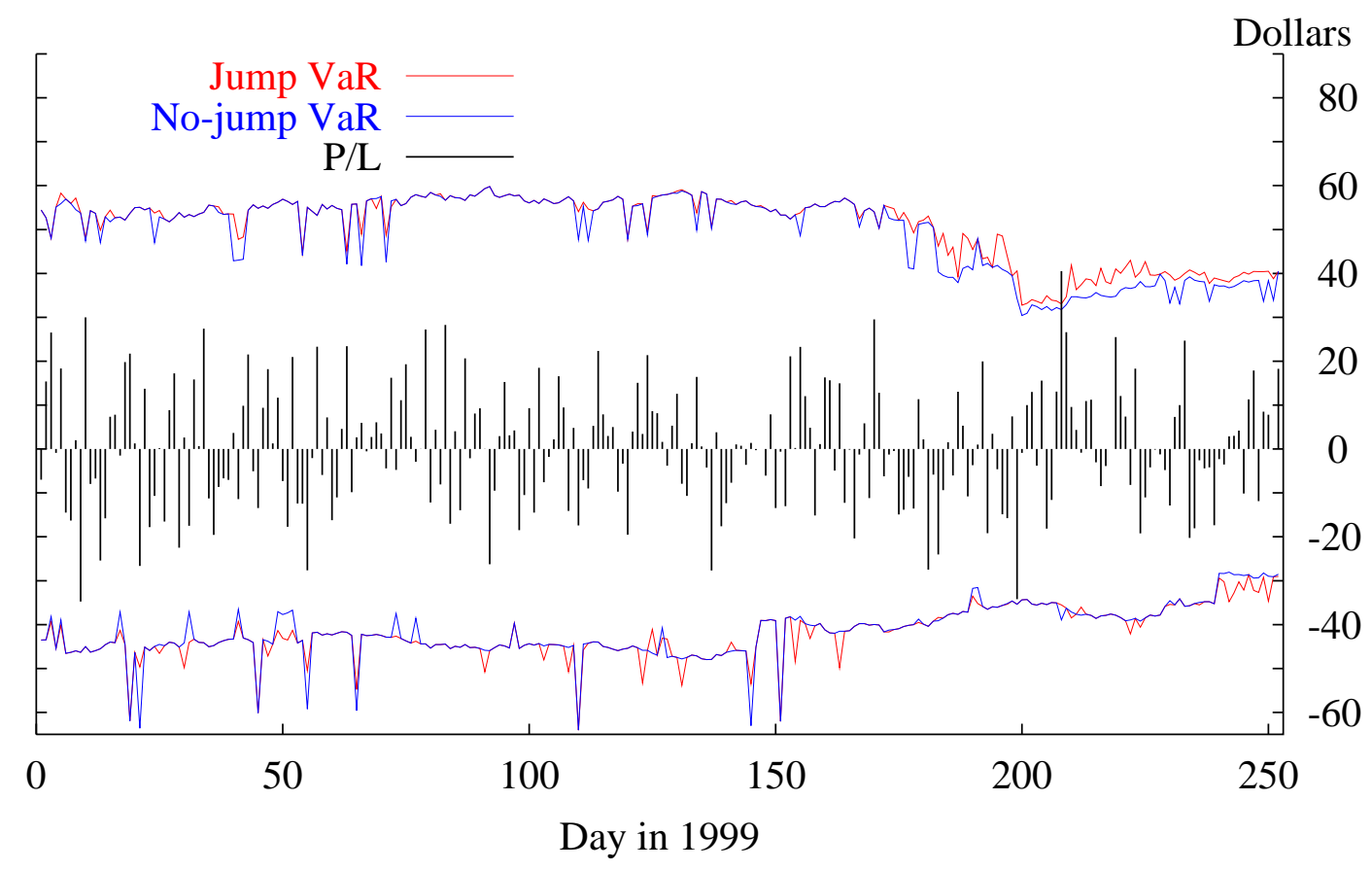

B. VaR estimated with the VCV model

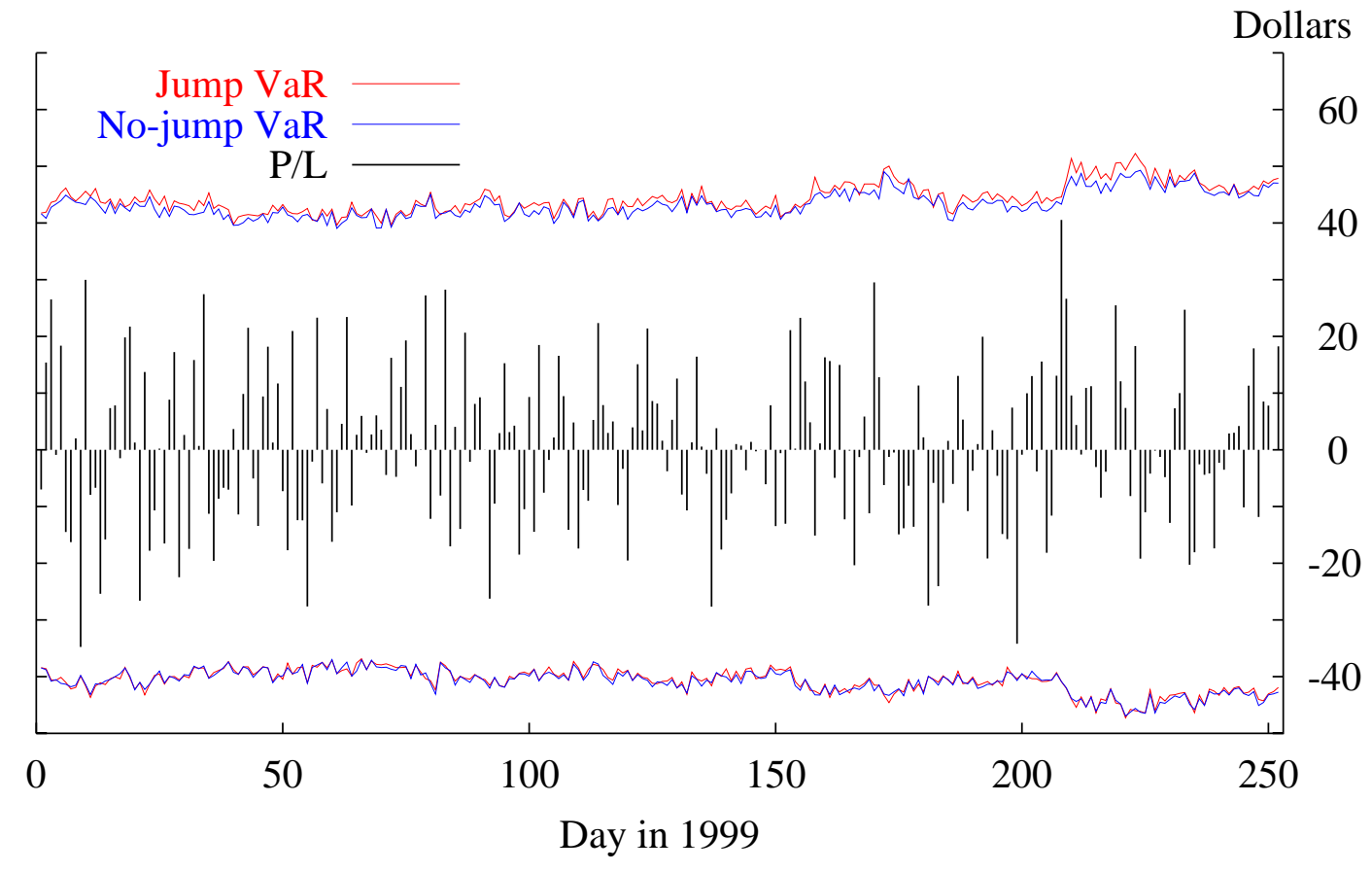


TABle 1. Common distributional assumptions in VAlue-AT-Risk models

Distributional assumption

Abbreviation

Historical simulation

HS

Exponentially-weighted historical simulation ${ }^{a}$

BRW

Filtered historical simulation ${ }^{b}$

FHS

Multivariate normal distribution with time-varying variance-covariance matrix (one example is the RiskMetrics ${ }^{\mathrm{TM}}$ model) ${ }^{c}$

VCV

${ }^{a}$ Boudoukh, Richardson, and Whitelaw (1998)

${ }^{b}$ Barone-Adesi, Giannopoulos, and Vosper (1999)

${ }^{c}$ J.P. Morgan/Reuters (1996) 
TABle 2. VAlue-AT-Risk estimates For AN InVEStMent OF \$100 In A Single LARGECAP STOCK ON JANUARY 4, 1999.

\begin{tabular}{lcc}
\hline \multirow{2}{*}{ Model } & \multicolumn{2}{c}{$\begin{array}{c}\text { VaR at indicated } \\
\text { percentile }(\$)\end{array}$} \\
\cline { 2 - 3 } & 95 percent & 99 percent \\
\hline HS & & \\
No jumps & 2.68 & 4.58 \\
With jumps & 2.80 & 6.84 \\
\hline VCV & & \\
No jumps & 3.31 & 4.68 \\
With jumps & 3.49 & 5.78 \\
\hline
\end{tabular}




\section{TABlE 3. ESTIMATED JUMP PARAMETERS FOR HISTORICAL SIMULATION}

The jump parameters are estimated as follows for each market capitalization group. Classify each daily return $r$ as follows:

$$
\begin{array}{cl}
r<-4 \sigma & \text { down jump } \\
-4 \sigma<r<4 \sigma & \text { no jump } \\
r>4 \sigma & \text { up jump }
\end{array}
$$

where $\sigma$ is the sample standard deviation of daily returns in this market cap group. The values of $\sigma$ are 1.88 percent for large-cap stocks, 2.40 percent for mid-cap stocks, and 4.05 percent for small-cap stocks. The jump probabilities $p$ and $q$ are estimated as the fraction of returns falling in the "down jump" and "up jump" groups, respectively. The jump sizes $D$ and $U$ are estimated as the mean of all returns in the "down jump" and "up jump" groups, respectively. In each market cap group, the jump parameters are estimated using approx-

\begin{tabular}{|c|c|c|c|}
\hline & Large cap & Mid cap & Small cap \\
\hline \multicolumn{4}{|c|}{ Parameter estimates } \\
\hline$p$ & .0020 & .0024 & .0027 \\
\hline$q$ & .0026 & .0037 & .0047 \\
\hline$D$ & .10 & .14 & .23 \\
\hline$U$ & .10 & .14 & .25 \\
\hline \multicolumn{4}{|c|}{ Expected number of days until next jump } \\
\hline Down jump & 500 & 417 & 370 \\
\hline Up jump & 385 & 270 & 213 \\
\hline
\end{tabular}
imately 4,000 firm-years of data (approximately 1 million daily returns) chosen at random from all firm-years present on the CRSP tape over 1980-99. 
Table 4. Scalar GARCH estimates on eight Sets of thirty EQuities

The scalar GARCH model with jumps is

$$
\begin{aligned}
& X_{t}=H_{t}^{1 / 2} \nu_{t}+\sum_{i=1}^{30} X_{J_{i}, t} \\
& H_{t}=S(1-\alpha-\beta)+\alpha X_{t-1} X_{t-1}^{\prime}+\beta H_{t-1} \\
& \nu_{t} \sim \mathbf{N}\left(0, I_{30}\right) \\
& X_{J_{i}, t}= \begin{cases}0 & \text { with probability } 1-p_{i}-q_{i}, \\
-D_{i} & \text { with probability } p_{i}, \\
U_{i} & \text { with probability } q_{i},\end{cases} \\
& p_{i}=\lambda \hat{p}_{i} \\
& q_{i}=\lambda \hat{q}_{i}
\end{aligned}
$$

with $\hat{p}_{i}, \hat{q}_{i}, D_{i}$ and $U_{i}$ as defined in Table 3 . The scalar GARCH model without jumps sets the $X_{J_{i}, t}$ terms equal to zero. Both models were estimated via maximum likelihood using the mixture model (5) on 8 different datasets. Each dataset consisted of 30 equities (10 large-cap, 10 mid-cap, and 10 small-cap).

\begin{tabular}{clllllll}
\hline & \multicolumn{3}{c}{ Without jumps } & & \multicolumn{3}{c}{ With jumps } \\
\cline { 2 - 3 } \cline { 7 - 8 } & Mean & Min & Max & & Mean & Min & Max \\
\hline$\alpha$ & 0.0093 & 0.0055 & 0.0126 & & 0.0127 & 0.0070 & 0.0209 \\
$\beta$ & 0.95 & 0.90 & 0.99 & & 0.94 & 0.91 & 0.99 \\
$\lambda$ & na & na & na & & 0.67 & 0.42 & 0.88 \\
Log-likelihood & 70.3 & 67.5 & 75.5 & & 71.8 & 68.3 & 76.4 \\
\hline
\end{tabular}


TABle 5. One-DAy 99 PERCENT VAR FOR THE LONG-ShORT PORTFOlio, ESTimated FOR THE 252 TRADING DAYS IN 1999

\begin{tabular}{|c|c|c|c|c|}
\hline & \multicolumn{2}{|c|}{ Mean VaR (\$) } & \multicolumn{2}{|c|}{ Number of violations } \\
\hline & Left tail & Right tail & Left tail & Right tail \\
\hline \multicolumn{5}{|l|}{ HS (Historical simulation) } \\
\hline No-jump model & -19.5 & 26.8 & 6 & 2 \\
\hline $\begin{array}{l}\text { Jump model not corrected } \\
\text { for double counting }\end{array}$ & -22.2 & 29.8 & 3 & 2 \\
\hline Jump-VaR model & -21.7 & 27.8 & 3 & 2 \\
\hline \multicolumn{5}{|l|}{ VCV (Scalar GARCH) } \\
\hline No-jump model & -19.0 & 20.2 & 5 & 4 \\
\hline $\begin{array}{l}\text { Jump model not corrected } \\
\text { for double counting }\end{array}$ & -21.6 & 22.9 & 2 & 4 \\
\hline Jump-VaR model & -20.8 & 22.0 & 3 & 4 \\
\hline
\end{tabular}


Table 6. One-DAy 99 Percent VAR For the long PortFolio, estimated for the 252 TRADING DAYS IN 1999

\begin{tabular}{|c|c|c|c|c|}
\hline & \multicolumn{2}{|c|}{ Mean VaR (\$) } & \multicolumn{2}{|c|}{ Number of violations } \\
\hline & Left tail & Right tail & Left tail & Right tail \\
\hline \multicolumn{5}{|l|}{ HS (Historical simulation) } \\
\hline No-jump model & -41.6 & 49.5 & 0 & 1 \\
\hline $\begin{array}{l}\text { Jump model not corrected } \\
\text { for double counting }\end{array}$ & -42.2 & 50.9 & 0 & 1 \\
\hline Jump-VaR model & -42.0 & 50.9 & 0 & 1 \\
\hline \multicolumn{5}{|l|}{ VCV (Scalar GARCH) } \\
\hline No-jump model & -40.9 & 43.3 & 0 & 0 \\
\hline $\begin{array}{l}\text { Jump model not corrected } \\
\text { for double counting }\end{array}$ & -41.4 & 45.4 & 0 & 0 \\
\hline Jump-VaR model & -40.7 & 44.3 & 0 & 0 \\
\hline
\end{tabular}


Table 7. Difference in one-Day 99 percent VaR Between the no-Jump and JUMP - VAR MODELS

\begin{tabular}{|c|c|c|c|c|}
\hline & \multicolumn{2}{|r|}{ Left tail } & \multicolumn{2}{|c|}{ Right tail } \\
\hline & $\begin{array}{l}\text { Dollar } \\
\text { amount }\end{array}$ & $\begin{array}{c}\text { As a percent } \\
\text { of no-jump VaR }\end{array}$ & $\begin{array}{c}\text { Dollar } \\
\text { amount }\end{array}$ & $\begin{array}{c}\text { As a percent } \\
\text { of no-jump VaR }\end{array}$ \\
\hline & \multicolumn{4}{|c|}{ LONG-SHORT PORTFOLIO } \\
\hline \multicolumn{5}{|l|}{ HS } \\
\hline Mean & 2.2 & 12 & 1.0 & 5 \\
\hline Maximum & 7.0 & 42 & 10.0 & 44 \\
\hline \multicolumn{5}{|l|}{ VCV } \\
\hline Mean & 1.8 & 10 & 1.8 & 10 \\
\hline \multirow[t]{2}{*}{ Maximum } & 3.3 & 20 & 3.9 & 16 \\
\hline & \multicolumn{4}{|c|}{ LONG PORTFOLIO } \\
\hline \multicolumn{5}{|l|}{ HS } \\
\hline Mean & 0.4 & 1 & 1.3 & 3 \\
\hline Maximum & 9.5 & 24 & 10.8 & 26 \\
\hline \multicolumn{5}{|l|}{ VCV } \\
\hline Mean & -0.2 & -0.4 & 1.0 & 2 \\
\hline Maximum & 1.3 & 3 & 3.4 & 8 \\
\hline
\end{tabular}


Table 8. Coverage Level of the no-Jump VaR model, ASsuming the Jump -VaR MODEL IS TRUE

\begin{tabular}{|c|c|c|}
\hline & Left tail & Right tail \\
\hline & \multicolumn{2}{|c|}{ LONG-SHORT PORTFOLIO } \\
\hline \multicolumn{3}{|l|}{ HS } \\
\hline Mean & 1.4 & 1.0 \\
\hline Maximum & 2.2 & 1.6 \\
\hline \multicolumn{3}{|l|}{ VCV } \\
\hline Mean & 1.6 & 1.6 \\
\hline \multirow[t]{2}{*}{ Maximum } & 2.1 & 2.2 \\
\hline & \multicolumn{2}{|c|}{ LONG PORTFOLIO } \\
\hline \multicolumn{3}{|l|}{ HS } \\
\hline Mean & 0.9 & 1.0 \\
\hline Maximum & 1.2 & 1.9 \\
\hline \multicolumn{3}{|l|}{ VCV } \\
\hline Mean & 1.0 & 1.2 \\
\hline Maximum & 1.2 & 1.4 \\
\hline
\end{tabular}


TABle 9. Ten-DAY 99 PERCEnt VAR For the LONG-Short PORTFolio, Estimated FOR THE 252 TRADING DAYS IN 1999

\begin{tabular}{cccccc}
\hline & \multicolumn{2}{c}{ Mean VaR $(\$)$} & & \multicolumn{2}{c}{ Number of violations } \\
& Left tail & Right tail & & Left tail & Right tail \\
\hline HS (Historical simulation) & & & & & 0 \\
No-jump model & -54.0 & 85.8 & & 0 & 0 \\
Jump-VaR model & -59.8 & 87.1 & & 0 & 0 \\
VCV (Scalar GARCH) & & & & & 0 \\
No-jump model & -56.7 & 70.7 & & 0 & 0 \\
Jump-VaR model & -64.4 & 79.2 & & 0 & 0 \\
\hline
\end{tabular}




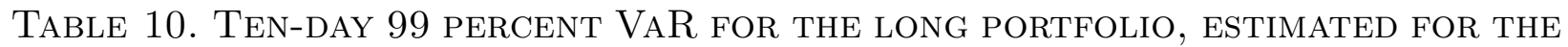
252 TRADING DAYS IN 1999

\begin{tabular}{cccccc}
\hline & \multicolumn{2}{c}{ Mean VaR $(\$)$} & & \multicolumn{2}{c}{ Number of violations } \\
\cline { 5 - 6 } & Left tail & Right tail & & Left tail & Right tail \\
\hline HS (Historical simulation) & & & & & \\
No-jump model & -114 & 158 & & 0 & 0 \\
Jump-VaR model & -112 & 166 & & 0 & 0 \\
VCV (Scalar GARCH) & & & & & 0 \\
No-jump model & -123 & 149 & & 0 & 0 \\
Jump-VaR model & -120 & 158 & & 0 \\
\hline
\end{tabular}


TABle 11. Comparison BetWeEn ACTUAL AND THEORETICAL Distribution of the NUMBER OF JUMPS PER DAY AMONG 90 RANDOMLY CHOSEN STOCKS OVER THE 504 TRADING DAYS IN 1998-99

\begin{tabular}{lccccc}
\hline \multirow{2}{*}{$\begin{array}{l}\text { Number of } \\
\text { jumps per day }\end{array}$} & $\begin{array}{c}\text { Number } \\
\text { of days }\end{array}$ & $\begin{array}{c}\text { Percent } \\
\text { of days }\end{array}$ & & $\begin{array}{c}\text { Number } \\
\text { of days }\end{array}$ & $\begin{array}{c}\text { Percent } \\
\text { of days }\end{array}$ \\
\cline { 2 - 3 } 0 & 206 & 40.9 & & 292 & 58.0 \\
1 & 165 & 32.7 & & 160 & 31.7 \\
2 & 67 & 13.3 & & 43 & 8.6 \\
3 & 27 & 5.4 & & 1.5 \\
4 & 16 & 3.2 & & 0.2 \\
5 or more & 23 & 4.6 & & 0 & $<0.1$ \\
Total & 504 & 100.0 & 504 & 100.0 \\
\hline
\end{tabular}

